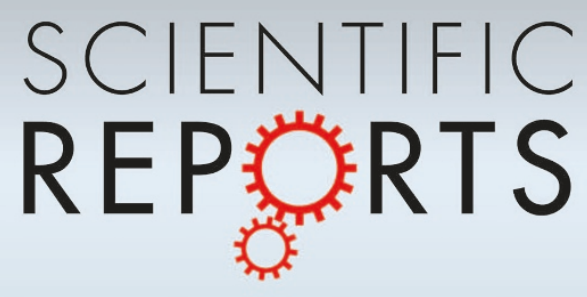

\title{
Defective folliculogenesis in female mice lacking Vaccinia-related kinase 1
}

SUBJECT AREAS:

IMAGING

BIOTECHNOLOGY

REPRODUCTIVE BIOLOGY

DEVELOPMENTAL BIOLOGY

Received

3 May 2012

Accepted

11 June 2012

Published

25 June 2012

Correspondence and requests for materials should be addressed to J.H.J. (jhje@postech. ac.kr) or K.T.K. (ktk@

postech.ac.kr)

* These authors contributed equally to this work.

\author{
Jinkyung Kim ${ }^{1,2 *}$, Yoon Ha Choi ${ }^{3 *}$, Soeun Chang ${ }^{1,4}$, Kyong-Tai Kim ${ }^{3,5}$ \& Jung Ho Je 1,2,4,6
}

\begin{abstract}
'X-ray Imaging Center, Pohang University of Science and Technology (POSTECH), Pohang, 70-784, South Korea, ${ }^{2}$ School of Interdisciplinary Bioscience and Bioengineering, Pohang University of Science and Technology (POSTECH), Pohang, 790-784, South Korea, ${ }^{3}$ Department of Life Science, Division of Molecular and Life Sciences, Pohang University of Science and Technology (POSTECH), Pohang, 790-784, South Korea, ${ }^{4}$ Department of Materials Science and Engineering, Pohang University of Science and Technology (POSTECH), Pohang, 790-784, South Korea, ${ }^{5}$ Division of Integrative Biosciences and Biotechnology, Pohang University of Science and Technology (POSTECH), Pohang, 790-784, South Korea, ${ }^{6}$ RIKEN SPring-8 Center, 1-1-1 Kouto, Sayo-cho, Sayo, Hyogo, 679-5198, Japan.
\end{abstract}

The Vaccinia-related kinase 1(VRK1), which is generally implicated in modulating cell cycle, plays important roles in mammalian gametogenesis. Female infertility in VRK1-deficient mice was reported to be caused by defective meiotic progression in oocyte at postovulatory stage. VRK1 roles in folliculogenesis, however, remain largely unknown. Here, accurate quantification of folliculogenesis is performed by a direct visualization of 'intact' ovary in 3-dimensions (3-D) using a synchrotron X-ray microtomography. In VRK1-deficient ovaries, the numbers of pre-antral and antral follicles are significantly reduced by $38 \%$ and $46 \%$, respectively, comparing to control. The oocytes volumes in antral and Graffian follicles also decrease by $42 \%$ and $37 \%$ in the mutants, respectively, indicating defects in oocyte quality at preovulatory stage. Genetic analysis shows that gene expressions related to folliculogenesis are down-regulated in VRK1-deficient ovaries, implying defects in folliculogenesis. We suggest that VRK1 is required for both follicle development and oocyte growth in mammalian female reproduction system.

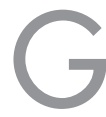

ametogenesis that is essential for the development of all sexually reproducing organisms requires the execution of several interrelated events including genetic exchange, haploidization, and cellular differentiation. Perturbations of the interrelated events result in dysfunction of male/female reproductive system and thus birth defects on their descendants ${ }^{1}$.

Vaccinia-related kinase 1 (VRK1), which is involved in modulating cell cycle progression ${ }^{2-5}$, is recently suggested as important player for gametogenesis. VRK1-deficient organisms show abnormality of reproductive organs, followed by defects on germ cell development. For example, VRK1-deficient Caenorhabditis elegans shows abnormal formation of the vulva, uterus, and utse. Studies in Drosophila melanogaster suggest that Nucleosomal histone kinase-1 (NHK-1), homologue of mammalian VRK1, acts as a downstream effector of the meiotic check-point in oocyte ${ }^{6}$. Very recently, infertilities in VRK1-deficient mice have been reported for both sexes $^{7-9}$. Specifically, VRK1-deficiency induces degeneration of testes resulted by progressive germ cell loss in male $^{7,8}$. Female infertility in mice lacking VRK1 was reported to be caused by post-ovulation defect, a delay in meiotic progression, which resulted in the presence of lagging chromosomes during formation of the metaphase plate $^{9}$. At preovulatory stage, however, there were no morphological differences between normal and VRK1deficient ovaries based on the ovary histology ${ }^{9}$. Here we argue that the possibility of pre-ovulation defects cannot be excluded only with the evidence of the histology data. To clarify this issue, accurate quantification of folliculogenesis in 'intact' ovary, instead of sectioned ovary, is required.

Accurate estimation of the number of follicles at various developmental stages is an important indicator of the process of folliculogenesis in relation to the endocrine signals and paracrine/autocrine mechanisms that control the growth and maturation of the oocytes ${ }^{10,11}$. In fact, follicle numbers can be significantly altered under impairment of reproductive system ${ }^{12,13}$. Previous approaches on the estimation of follicular numbers per ovary, mostly based on several optical/physical sections, however, have considerable potential for quantitative errors and bias $^{10,14,15}$.

Here we present an alternate strategy of synchrotron X-ray microtomography that can visualize the morphologies of follicles in intact ovaries and give access to quantitative analysis in 3-dimensions (3-D) geometry. Phase contrast based on strongly collimated synchrotron X-rays ${ }^{16-18}$ produces images of excellent quality due to strong 

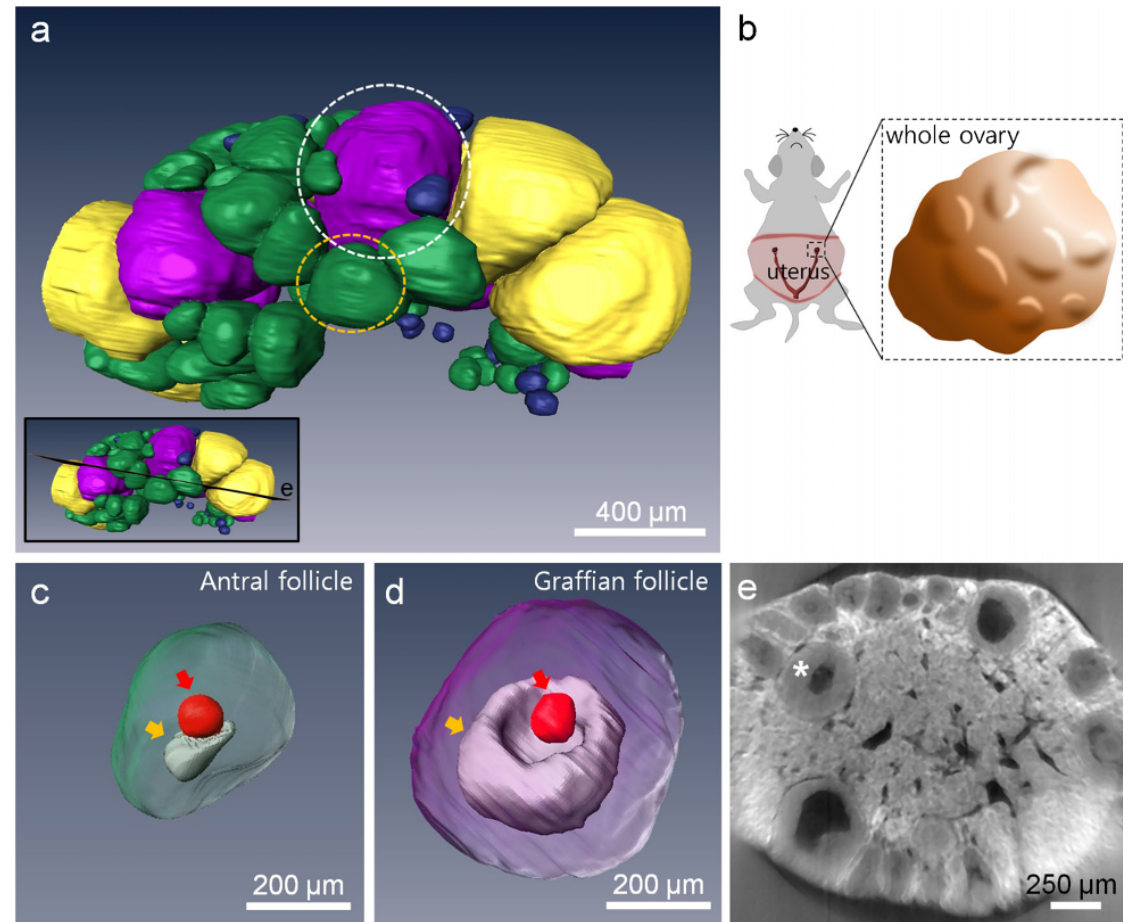

Figure 1 3-D segmented images of a normal mouse ovary. (a) Representative segmented image (Supplementary Movie 2) of a whole ovary in 6-weekold normal mouse. navy, pre-antral follicle; green, antral follicle; violet, Graffian follicle; yellow, corpus luteum. (b) Schematic diagram of a whole ovary in mouse. (c) Transparent image of an antral follicle (yellow dashed circle in Fig. 1a; Supplementary Movie 3). (d) Transparent images of a Graffian follicle (white dashed circle in Fig. 1a; Supplementary Movie 4). yellow arrows in (c) and (d), antrum cavity; red arrows in (c) and (d), oocytes. (e) Slice image in a given plane (inset of Fig. 1a).

edge enhancement between different regions ${ }^{19}$. Furthermore highpenetration power of synchrotron X-rays allows us to analyze thick specimens with high resolution, as required here $\mathrm{e}^{20}$.

\section{Results}

3-D microarchitecture of whole ovaries in normal mice. Quantifying microstructure over a whole organ could provide a useful map of anatomical structure with the potential to give functional insight ${ }^{21,22}$. We first performed microtomography for whole ovaries (Fig. 1b) in normal mice, as representatively demonstrated by the 3-D volume rendered image of an ovary in Supplementary Movie 1. Follicles and corpus lutea (CLs) were segmented based on the volume rendered image, as demonstrated in the 3-D image of Fig. 1a (Supplementary Movie 2). Pre-antral (navy), antral (green), Graffian (violet) follicles as well as CLs (yellow) are clearly resolved in 3-D geometry in the whole ovary.

The 3-D transparent images of the antral follicle in Fig. 1c (yellow dashed circle in Fig. 1a; Supplementary Movie 3) and the Graffian follicle in Fig. 1d (white dashed circle in Fig. 1a; Supplementary Movie 4) clearly reveal that the oocyte (red arrow in Fig. 1c or d) shows no significant difference in size. The antrum cavity size (yellow arrow in Fig. 1c or d) is, however, much smaller in the antral than in the Graffian follicle, as well known.

All the follicles and CLs are densely distributed in the ovarian cortex, as demonstrated in Fig. 1a and Supplementary Movie 2. Remarkably they are positioned in contact with the ovarian surface epithelium, except a few pre-antral follicles. This is not clear in the 2$\mathrm{D}$ slice image of Fig. 1e in a given plane (see inset of Fig. 1a). For example, the Graffian follicle, marked by an asterisk in Fig 1e in a given plane (see inset of Fig. 1a), is in reality in contact with the surface (Supplementary Movie 2).

3-D microarchitecture of whole ovaries in VRK1-deficient mice. Figure 2a (Supplementary Movie 5) shows a representative segmented image for a whole ovary in a VRK1-deficient mouse, based on the volume rendered image of Supplementary Movie 6. The VRK1-deficient ovary also shows the same stages of follicles in folliculogenesis as the normal ovary in Fig. 1a. However, the volume fraction of the stroma is increased from $56 \%$ to $64 \%$, comparing to the normal ovary (Supplementary Movies 2 and 5). Figure $2 b$ and $c$ show the 3-D transparent images of the antral follicle (yellow dashed circle in Fig. 2a; Supplementary Movie 7) and the Graffian follicle (white dashed circle in Fig. 2a; Supplementary Movie 8). We note that the sizes of the oocytes are smaller than those in normal follicles.

Very interestingly we directly observe a ruptured follicle without oocyte in the 3-D image of Fig. 2b (Supplementary Movie 9), a different view (red arrow head) of the follicle (a white dashed circle) in Fig. 2a. In fact such a ruptured follicle was observed 11 times in 6 VRK1 deficient ovaries and 4 times in 3 normal ovaries. We note that the granulosa layer of this follicle is very thin as $11 \mu \mathrm{m}$ (see Fig. $2 \mathrm{c}$, the yellow dashed plane of Fig. 2b), comparing to typical Graffian follicle with a granulosa layer of $85 \mu \mathrm{m}$ in Fig. 2d, which is the slice image of the follicle marked by an asterisk in Fig. $2 \mathrm{a}$. This result confirms that ovulation successfully occurs even in VRK1-deficient mice.

Effects of VRK1-deficiency on follicle number and volume. For statistical analysis, we measured the number of follicles and CL for whole ovaries in normal and VRK1-deficient mice ( $n=3$ /genotype) with various ages (age 6, 16, 43 weeks) (Fig. 3; Table 1). The segmented 3-D image of whole ovary without any optical/physical sectioning allows us to precisely quantify the numbers of follicles and CL. The numbers of pre-antral ( $\geq 50 \mu \mathrm{m}$ in diameter), antral, and Graffian follicles, and of CL per an ovary for the normal mice in Table 1 would be a baseline for comparison with those of genetically modified animals. For the VRK1-deficient ovaries at 6 weeks, we find that the numbers of pre-antral and antral follicles are significantly reduced as $36 \%$ and $47 \%$, respectively, but with little changes in those of Graffian follicle and CL (Fig. 3a; Table 1). 

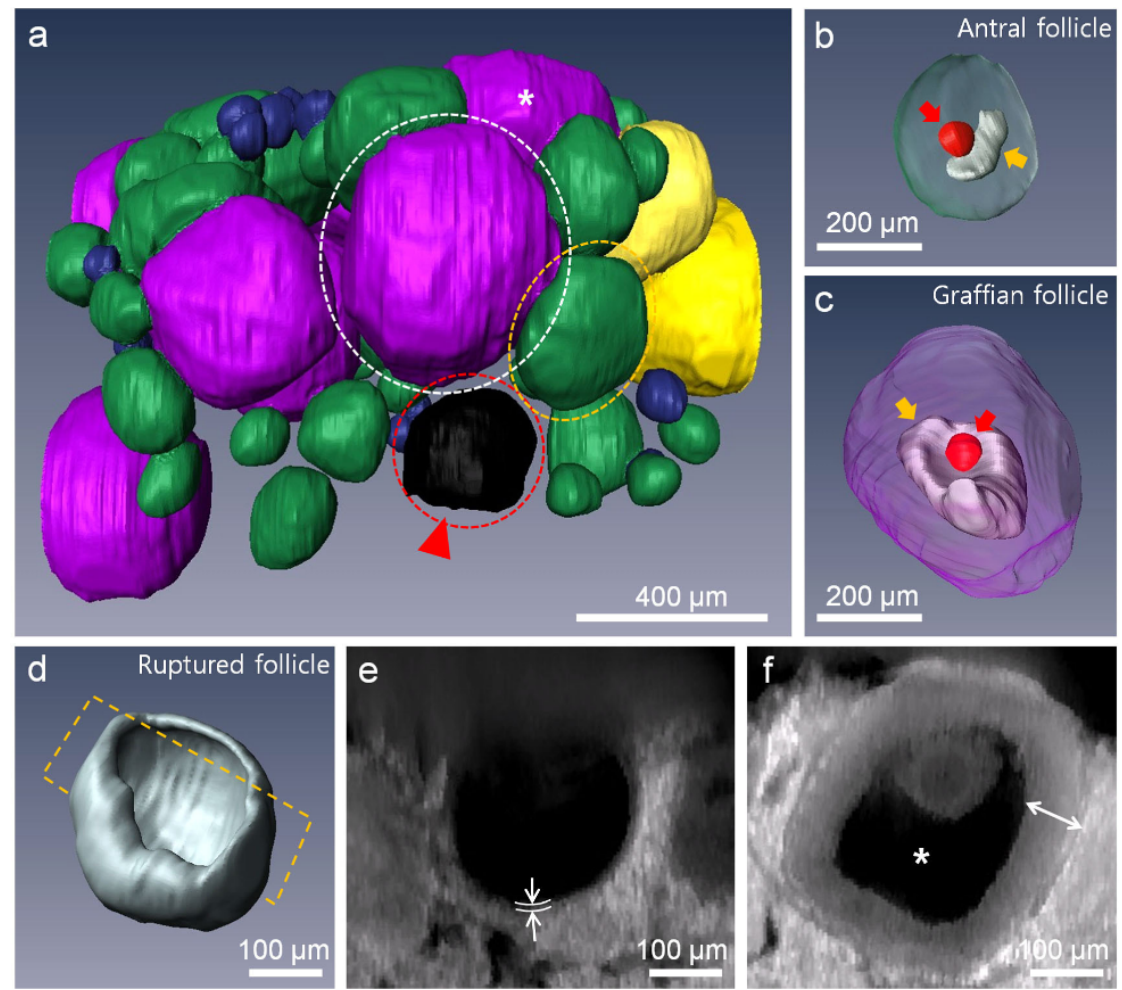

Figure 2 3-D segmented images of a VRK1-deficient mouse ovary. (a) Representative segmented image (Supplementary Movie 5) of a whole ovary in 6-week-old VRK1-deficient mouse. navy, pre-Antral follicle; green, antral follicle; violet, Graffian follicle; yellow, corpus luteum; black; Ruptured follicle. (b) Transparent image of an antral follicle (yellow dashed circle in Fig. 2a; Supplementary Movie 7). (c) Transparent images of a Graffian follicle (white dashed circle in Fig. 2a; Supplementary Movie 8). yellow arrows in (b) and (c), antrum cavity; red arrows in (b) and (c), oocytes. (d) Segmented image of the ruptured follicle, a different view (red arrow head) of the follicle (a white dashed circle) in Fig. 2a (Supplementary Movie 9). (e) Slice image of the ruptured follicle (yellow dashed plane in Fig. 2d). (f) Slice image of the Graffian follicle (asterisk of Fig. 2a). arrows in (e) and (f), granulosa layer with thicknesses as $11 \mu \mathrm{m}$ and $85 \mu \mathrm{m}$, respectively.
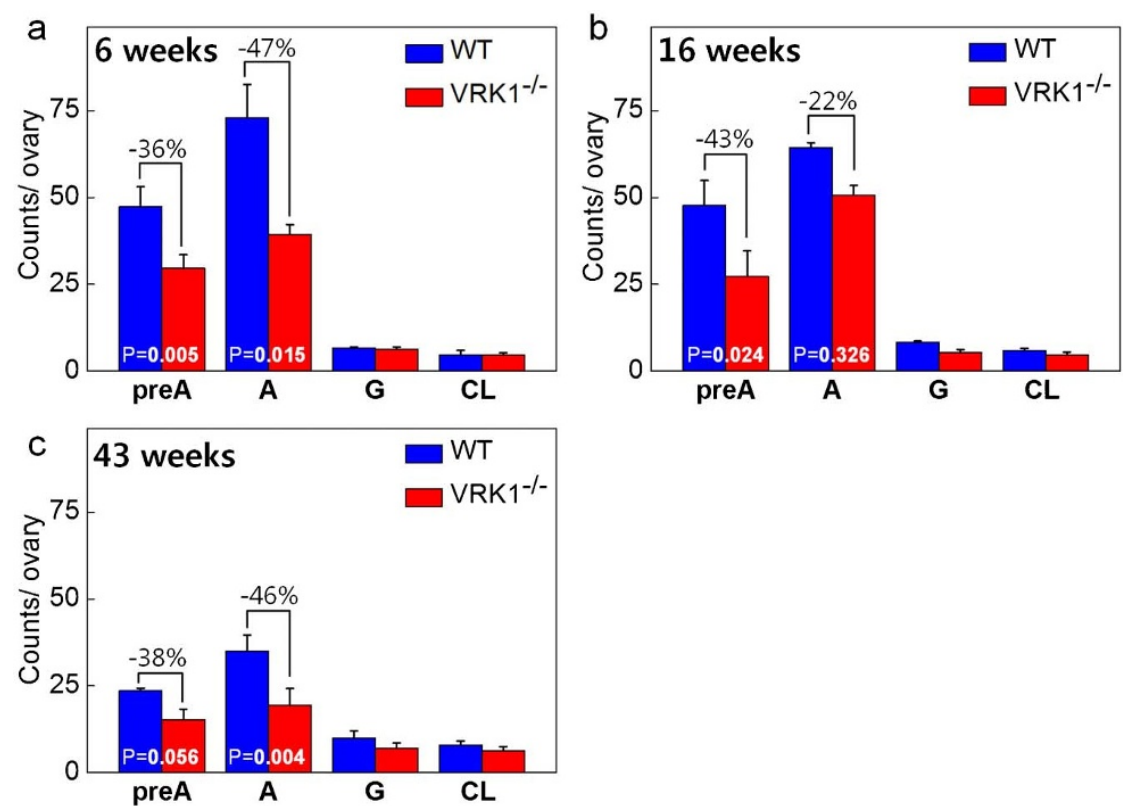

Figure 3 Effect of VRK1-deficiency on follicle and CL numbers. (a) Comparison of average numbers of follicles and CL per ovary in normal and VRK1deficient mice with 6 weeks of ages ( $n=3$ /genotype). The errors correspond to the s.e.m.. (b) and (c) Comparison of the numbers per ovary for 16-weekold and 43-week-old mice, respectively ( $n=3$ /genotype). preA, pre-Antral follicle; A, anntral follicle; $G$, Graffian follicle; CL, corpus luteum; P, p-value. 
Table 1 | The number of follicles and CL per ovary in normal and VRK1-deficient mice with various ages

\begin{tabular}{|c|c|c|c|c|c|}
\hline age(weeks) & genotype & \multicolumn{3}{|c|}{ Follicle no./ovary } & $\begin{array}{c}\text { CL no./ovary } \\
\text { Corpus lutea }\end{array}$ \\
\hline 6 & Normal & $47 \pm 6$ & $73 \pm 10$ & $7 \pm 1$ & $5 \pm 1$ \\
\hline \multirow[t]{2}{*}{16} & Normal & $47 \pm 7$ & $64 \pm 1$ & $8 \pm 1$ & $6 \pm 1$ \\
\hline & VRK $1^{-/-}$ & $27 \pm 7$ & $50 \pm 3$ & $5 \pm 1$ & $5 \pm 1$ \\
\hline 43 & Normal & $24 \pm 1$ & $35 \pm 5$ & $10 \pm 2$ & $8 \pm 1$ \\
\hline
\end{tabular}

Values are the mean \pm SEM for three ovaries in three mice.

This trend is similarly observed even in the mice at 16 and 43 weeks (Fig. 3b and c; Table 1). The significant decrease in the numbers of pre-antral (49\%) and antral (52\%) follicles in the 43-week-old normal mice, comparing to 6-week-old normal mice (blue bars in Fig. $3 a, c)$, is conceivable from reduced fertilization capacity in advanced age.

We also measured the follicle volumes accurately at various stages and by age/genotype, as illustrated in Supplementary Table 1. There is no significant difference by age or genotype, indicating no correlation between follicle volume and age/genotype.

Effect of VRK1-deficiency on oocyte volume. Oocyte size is associated with oocyte quality or meiotic competence ${ }^{11,23,24}$. For verifying oocyte quality in VRK1-deficient mice, we directly measured the oocyte volumes from the segmented images in normal and VRK1-deficient ovaries ( $n=6 /$ genotype) at various ages (age 6, 16, 43 weeks) (Fig. 4; Table 2): the analyzed oocytes were only the ones in antral and Graffian follicles. This quantification detected further interesting differences between normal and VRK1-deficient oocytes, not immediately evident from the images. We present here that the oocyte volumes in antral and Graffian follicles show no significant difference as $3.37 \times 10^{5} \pm 0.13 \times 10^{5} \mu \mathrm{m}^{3}$ (mean $\pm \mathrm{SEM}$ )

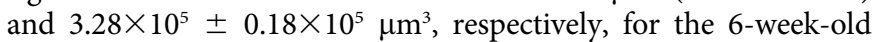
normal mice (Fig. 4a, b; Table 2). The oocyte volumes show no considerable differences by ages $(6,16$, and 43 weeks) as well, as seen in Fig. $4 \mathrm{a}$ and $\mathrm{b}$.

Remarkably, the oocyte volumes are significantly reduced in VRK1-deficient mice, for instance, by $42 \%$ in antral (Fig. $4 \mathrm{a}$ ) and $37 \%$ in Graffian follicles (Fig. 4b), respectively, for 6 weeks age. Similar reductions in the oocyte volumes are observed for the mice at later ages (16 and 43 weeks of ages) (Fig. 4a, b). This result suggests that oocyte quality is impaired at preovulatory stage in VRK1-deficient mice, possibly explaining another cause for their infertility in addition to the postovulatory defects'.

To study possible association of VRK1 to antrum formation, we directly measured the volume of antrum cavity in Graffian follicles at various ages. Supplementary Fig. S1a shows the schematic picture of a Graffian follicle with the oocyte (black), the granulosa/cumulus cells (orange), and the antrum cavity. Interestingly, the volume of the antrum cavity in Graffian follicle is not altered by genotypes at all ages (Supplementary Fig. S1b), suggesting that VRK1 may not play a critical role in antrum formation during folliculogenesis.

Effects of VRK1-deficiency on different pathways involved in folliculogenesis. For analyzing different pathways involved in folliculogenesis in mice lacking VRK1, we examined gene expression patterns in follicle cells from normal $(n=3)$ or VRK1deficient ovaries $(n=4)$, as demonstrated in Fig. 5. Primary, preantral, and antral/Graffian follicles were isolated from the ovaries of both genotypes and analyzed by qRT-PCR method. We note that the expression levels of 17 genes involved in both early (Fig. 5a) and later (Fig. 5b) folliculogenesis are significantly reduced in VRK1-deficient mice. This implies that VRK1 deficiency results in defects in folliculogenesis from early to later stages.

\section{Discussion}

This study provides accurate, 3-D quantification of folliculogenesis in normal and VRK1-deficient mice by a direct visualization of 'intact' whole ovaries using a synchrotron X-ray microtomography.

Study on follicular number can provide important information about the function of the ovary, specifically the relationship between folliculogenesis and the factors that regulate the survival and maturation of follicles at any stage of their development ${ }^{10}$. Changes in follicle numbers have been reported in model mice with defective reproductive function ${ }^{25,26}$. In this study, the follicle numbers at various stages for normal whole ovaries could be a baseline for comparison with those of genetically modified animals. Notably the significant reduction of the follicle numbers in VRK1-deficient ovaries implies defects in follicle development, leading to infertility. This result may contribute to understanding of VRK1 function at preovulatory stage in female reproductive system.

Mammalian oocytes have been proposed to have important roles in the orchestration of ovarian follicular development and
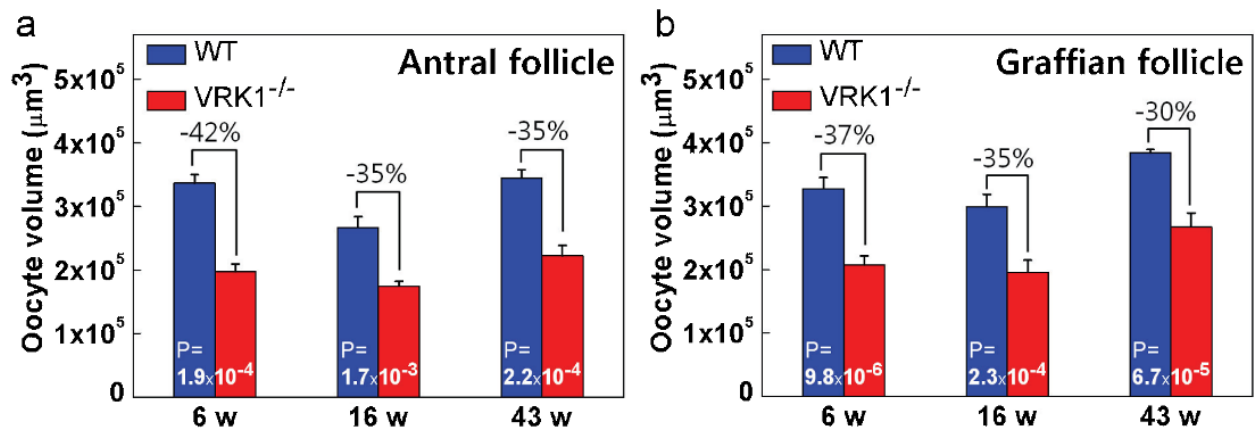

Figure 4 Effect of VRK1-deficiency on oocyte volume. Comparison of average oocyte volumes in antral (a) and Graffian (b) follicles in normal and VRK1-deficient mice with various ages $(6,16,43$ weeks). The errors correspond to the s.e.m. $(n=6)$. P, p-value. 
Table 2 | The oocyte volumes in antral and Graffian follicles in normal and VRK 1-deficient mice with various ages

Oocyte volume $\left(\mathrm{X} 10^{5} \mu \mathrm{m}^{3}\right)$

\begin{tabular}{clccc}
\hline & genotype & 6 weeks & 16 weeks & 43 weeks \\
\hline antral & Normal & $3.37 \pm 0.13$ & $2.67 \pm 0.16$ & $3.45 \pm 0.13$ \\
follicles & VRK1 ${ }^{-1-}$ & $1.97 \pm 0.13$ & $1.74 \pm 0.08$ & $2.23 \pm 0.16$ \\
Graffian & Normal & $3.28 \pm 0.18$ & $3.00 \pm 0.20$ & $3.84 \pm 0.06$ \\
follicles & ${\text { VRK } 1^{-1-}}^{l} 2.07 \pm 0.15$ & $1.96 \pm 0.19$ & $2.67 \pm 0.22$ \\
\hline \multicolumn{7}{l}{ Values are the mean \pm SEM for six oocytes. }
\end{tabular}

fertility ${ }^{27,28}$. Oocyte quality is acquired during folliculogenesis as the oocyte grows and during the period of oocyte maturation ${ }^{29}$. By clear relationships between the oocyte quality and size ${ }^{24,30-32}$, the comparison of oocyte size is often used as a marker for oocyte quality or meiotic competence. In fact, oocytes in experimentally altered ovaries are retarded in their physical growth in size $e^{25,26,33}$. However, invitro methods based on hyperstimulation or maturation in plate have considerable potential for quantitative errors and bias in the oocyte size measurement. The direct visualization of intact whole ovary in this study enabled us to accurately quantify the oocyte size, specifically, the oocyte volume, without optical/physical sectioning. The oocyte volumes in VRK1-deficient follicles are much smaller than those in normal follicles. The volume reduction in VRK1-deficient ovaries suggests that the VRK1 is required at a specific stage in oogenesis. Meanwhile, no significant changes in the oocyte volume between antral and Graffian follicles in normal mice is consistent with a previous report about oocyte diameter ${ }^{11}$.

Previous report on VRK1 role in mammalian oogenesis suggested that reduction of VRK1 activity causes a delay in meiotic progression during oogenesis, results in the presence of lagging chromosomes during formation of the metaphase plate, and ultimately leads to the failure of oocytes to be fertilized'. Besides the VRK1 role at 'postovulatory' stage in the previous report, we successfully reveal the role of VRK1 at 'preovulatory' stage, i.e. during folliculogenesis, from accurate quantification based on 'intact' ovary microtomography.

For analyzing different pathways involved in folliculogenesis in mice lacking VRK1, we examined the expression patterns of genes that are related to folliculogenesis. The down-regulations of the following gene expressions in VRK1-deficient ovaries (Fig. 5) could support the significant reductions in the pre-antral and antral follicle numbers in Fig. 3. Specifically the reduced expression levels of factor in the germline alpha (FIG $\alpha$ ) and anti-müllerian hormone (AMH) indicate defects in primordial follicle formation ${ }^{34-36}$. Down-regulated connexin 43 (CX43) also supports the follicle number reductions, since phenotype of CX43-deficient mouse showed the reduction in the germ cell number ${ }^{37}$. The decrease in kit receptor (KIT) and kit ligand (KITL) levels, since they are known to regulate the initiation of follicle growth and preantral follicular development ${ }^{38,39}$, could contribute to the follicle number reductions as well. Notably, the expression of growth differentiation factor-9 (GDF9) significantly reduces by $64 \%$ in pre-antral follicles of VRK1-deficient ovary. In GDF9deficient mouse, folliculogenesis is arrested at the one-layer follicle stage $^{40,41}$. So the low level of GDF9 in VRK1-deficient mice in our study possibly implies blocking normal follicular development, resulting in the follicle number reduction. VRK1 deficiency also reduces the levels of follicle-stimulating hormone receptor (FSHR) and superoxide dismutase 1 (SOD1) that play roles in antrum formation $^{42-44}$, again indicating the follicle number reductions. Taken together, these down-regulated gene expressions which function in early folliculogenesis support VRK1 role in follicle development. Here we cannot rule out the possibility that VRK1-deficiency can induce the follicular atresia in early folliculogenesis by the activation of apoptosis-related signal pathways. In fact VRK1-deficiency resulted in severe apoptosis, followed by a progressive loss of germ cells in male mice ${ }^{7,8}$. The relationship between VRK1 and survival of follicles needs to be clarified.

The down-regulations of wingless-related MMTV integration site 4 (Wnt4) and discoidin domain receptor 2 (DDR2) expressions in Fig. 5 emphasize the defected oocyte quality in VRK1-deficient ovary. Wnt 4 expression is important in oocyte maintenance ${ }^{45}$ while DDR2 is known as an important factor for follicle maturation ${ }^{46,47}$.

Steroid hormone action is also critical for ovarian function and female fertility. In VRK-deficient mouse, the hormone related genes are down-regulated (Fig. 5), indicating abnormal regulation of hormone level. For instance, the expressions of estrogen receptor $\beta$ $(E R \beta)^{48}$ is reduced in the mutant mice. Here no significant change of estrogen receptor $\alpha(E R \alpha)$ expression level between genotypes in Fig. 5b implies that VRK1 affects ER $\beta$ signaling rather than $E R \alpha^{4,50}$. The level of testicular orphan nuclear receptor 4 (TR4) which can
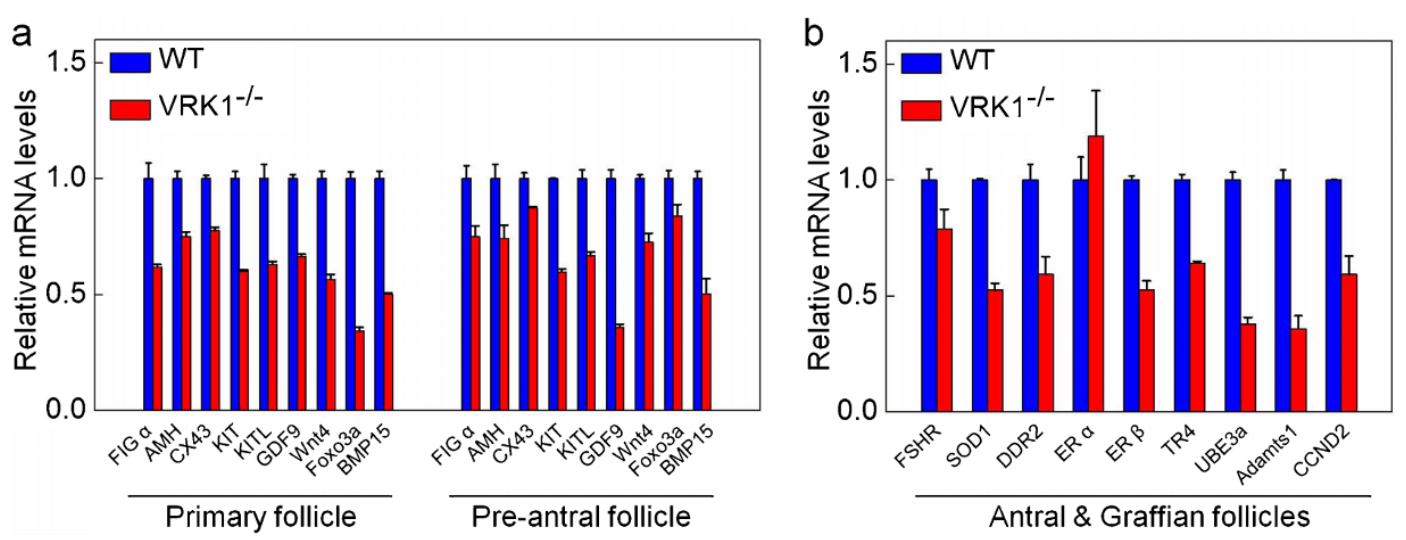

Figure $5 \mid$ Effects of VRK1-deficiency on different pathways involved in folliculogenesis. (a) Several marker genes, expressed in early folliculogenesis, were detected in primary and pre-antral follicles from whole ovaries of normal or VRK1-deficient mice by qRT-PCR. (b) Several marker genes, expressed in later folliculogenesis, were detected in antral/Graffian follicles from whole ovaries of normal or VRK1-deficient mice by qRT-PCR. Each value of qRTPCR was normalized to $\beta$-actin expression levels and expressed as the fold change relative to the levels detected in normal ovaries, which were set equal to 1. The errors correspond to the s.e.m.. FIG $\alpha$, Factor in the germline alpha; AMH, anti-müllerian hormone; CX43, Connexin 43; KIT, Kit receptor; KITL, Kit ligand; GDF9, Growth differentiation factor-9; Wnt4, Wingless-related MMTV integration site 4; Foxo3a, Forkhead box O3A; BMP15, Bone morphogenic protein15; FSHR, FSH receptor; SOD1, Superoxide dismutase 1; DDR2, Discoidin domain receptor 2; ER $\alpha$, Estrogen receptor $\alpha$; ER $\beta$, Estrogen receptor $\beta$; TR4, Testicular orphan nuclear receptor 4; UBE3a, Ubiquitin protein ligase E3A; ADAMTS1, ADAM-metallopeptidase with thrombospondin type 1 motif-1; CCND2, Cyclin D2. 
directly regulate luteinizing hormone receptor (LHR) gene expression $^{51}$ is reduced. Ubiquitin protein ligase E3A (UBE3a), that is known to function as a coactivator of steroid receptor-dependent gene expression $^{52}$, also shows low expression level. The expression of $\mathrm{AMH}$ that is known as an inhibitory endocrine factor for FSH action is down-regulated as well ${ }^{53}$. Therefore, we suggest that the maturation of follicles is retarded by incomplete hormone signaling in VRK1-deficient ovary.

Oocyte-somatic cell crosstalk is critical for the release of a fertilizable egg ${ }^{27}$. We find that the expressions of the genes which are related to somatic cells in folliculogenesis are reduced in VRK1-deficient ovaries (Fig. 5). For instance, the expression of forkhead box O3a (Foxo3a) that is known to coordinate between oocytes and granulosa cells ${ }^{54}$ is down-regulated in the mutant mice. Bone morphogenic protein15 (BMP15), oocyte specific homology of GDF9, that plays a role in cumulus-oocyte complex (COC) formation ${ }^{55}$ shows low expression level. The expression of ADAM-metallopeptidase with thrombospondin type 1 motif- 1 (ADAMTS1) that is important for morphogenesis of follicle wall and COC matrix for successful ovulation and fertilization ${ }^{56}$ is down-regulated as well. The level of cyclin D2 (CCND2) that functions in granulosa cell proliferation ${ }^{57}$ is also reduced.

Despite the reduced expressions of the genes which are related to both antrum formation and morphogenesis of the somatic cells in the VRK1 deficient mice (Fig. 5b), we observed no noticeable phenotypic difference in Graffian follicles (Supplementary Fig. S1b). Note that the antrum cavity was measured only for Graffian follicles (Fig. S1b) while the genes were detected in antral/Graffian follicles (Fig. 5b). Thus it is not straightforward to correlate the reduction of the gene expression with the antrum cavity size of Graffian follicles. Here we cannot rule out the possibility that the degree of the gene expression changes might be insufficient to induce the structural changes. Or other genes might be also involved in the antrum formation in VRK1 deficient mice. Further experiments will be required to investigate these possibilities in VRK1 deficient mice.

Overall, the direct visualization of 'intact' whole ovaries in 3-D using a synchrotron X-ray imaging method enabled us to quantify follicles at various stages in folliculogenesis, including oocytes in antral and Graffian follicles. Our accurate, quantitative results, supported by genetic analysis, could suggest possible roles of VRK1 in mammalian female reproductive system and provide another potential causes for infertility of VRK1-deficient mice in addition to the postovulatory defects ${ }^{9}$.

\section{Methods}

Ethics Statement. Approval of the study protocol was obtained from the Pohang University of Science and Technology Institutional Animal Care and Use Committee (approval ID: LIFE 012). All animal experiments were carried out according to the provisions of the Animal Welfare Act, PHS Animal Welfare Policy, and the principles of the NIH Guide for the Care and Use of Laboratory Animals. All mouse lines were maintained under specific pathogen-free conditions at the POSTECH animal facility under institutional guidelines.

Mice. The gene-trapped ES cell line RRR178 was obtained from BayGenomics. This cell line was generated using a gene trap protocol with the pGT1lxf construct, which contains the intron from engrailed 2 upstream of a gene encoding $\beta$-galactosidase/ neomycin resistance, $\beta$-geo (see http://www.genetrap.org). The ES cell clone was injected into a C57BL/6 blastocyst according to standard procedures. Male chimeras were bred with $\mathrm{C} 57 \mathrm{BL} / 6$ mice to create animals with a germ-line transmission of the mutant allele. Heterozygous mice were backcrossed a minimum of six generations with C57BL/6 mice prior to the study. Genotyping was performed by PCR and Southern blot using DNA extracted from mouse tails. Insertion of the $\beta$-geo cassette was verified by PCR ( $\beta$-geo primer sense, 5'ATCGCAGATCTGGACTCTAGAGGATCC- $3^{\prime}$ and antisense, $5^{\prime}$-ATGCGCTCAGGTCAAATTCAGACGGCAA- $3^{\prime}$ and Int 4 primer sense, $5^{\prime}$-ATCGCAGATCTGGACTCTAGAGGATCC- $3^{\prime}$ and antisense, 5' -GGAGAAACTTTGTACAGCTTCGTT- 3' , 5' AAGGAATCTTGGTTAGCTTTCAGA-3').

Tissue preparation. The 18 mice ( $n=3$ /genotype; age 6,16 , and 43 weeks) were perfused with phosphate buffered saline (PBS) and $4 \%$ paraformaldehyde (PFA). Ovaries were postfixed with $1 \%$ osmium tetroxide for 1 hour at $4{ }^{\circ} \mathrm{C}$. After standard dehydration in ethanol series, the samples were embedded in Epon 812 (epon 812 kit: EMS, Hatfield, PA, USA) for the observation by synchrotron X-ray imaging.

Synchrotron X-ray imaging. The microradiographic experiments were performed at BL29XU RIKEN Coherent X-ray Optics beamline (SPring-8, Japan: http:// www.spring8.or.jp/en). The monochromatic synchrotron X-ray was produced by a double crystal monochromator, and then transported into experimental hutches. In order to block or control the X-ray flux, a mechanical shutter with a Pt-Ir blade or Si attenuators was used respectively. Sample was mounted on a high precision motorcontrolled stage with rotational, tilting, and translational resolutions of $0.002^{\circ}$, $0.0009^{\circ}$, and $250 \mathrm{~nm}$, respectively. After passing through the sample, the transmitted $\mathrm{X}$-rays were converted to visible image using a scintillator $\left(\mathrm{CdWO}_{4}\right.$ : Nihon Kessho Koogaku Co. Ltd., Hinata Tatebayashi-City Gunma, Japan). After reflected by a mirror and magnified by an optical lens, the image was captured by a CCD camera.

3-D images acquisition and reconstruction. The microtomographic experiments for the visualization in 3-D were carried out by taking 1000 microradiographs at every $0.18^{\circ}$ rotation step, calibrated with background images. The projected image set was reconstructed with the standard filtered backprojection reconstruction algorithm using the Octopus8.5 software ${ }^{58}$ (inCT, Zwijnaarde, Belgium). Reconstructed slices consisted of $1600 \times 1600$ pixels in the horizontal and vertical directions. Volumerendered $3 \mathrm{D}$ images were obtained from the vertically stacked $2 \mathrm{D}$ slices using the Amira5.2 software (Visage Imaging, San Diego, CA, USA).

Quantitative analysis. For 3-D quantitative analysis, reconstructed image stacks of follicles and CLs in whole ovaries were manually segmented (Amira5.2 software). Here the segmentation of pre-antral follicles was done only for the ones larger than $50 \mu \mathrm{m}$ in diameter. The main criteria to classify development stages of follicles are the follicle size, the presence and size of antrum cavity, and the oocyte position within the follicle ${ }^{11}$. The CL could be easily identified by lower contrast than follicles.

Statistical analysis. To calculate the statistical significance in the difference between normal and VRK1 deficient ovaries, we applied the right-tail two sample t-test ${ }^{59}$ to the follicle numbers (or oocyte volumes) measured from three (or six) independent replicates. In this right tail t-test, we used the null hypothesis that the normal ovaries have larger follicles (eggs) than VRK1 deficient ovaries and the unequal variance assumption.

Quantitative RT-PCR. Ovarian follicles from normal $(n=3)$ or VRK1-deficient ovaries $(n=4)$ were mechanically isolated using fine forceps under dissecting microscope. Total RNA samples were prepared from intact follicle cells using Trizol (Invitrogen, Carlsbad, CA, USA) and then reverse transcribed using the ImProm-II Reverse Transcription System (Promega, San Luis Obispo, CA, USA): primary and pre-antral follicles from 2 week-old mice; antral and Graffian follicles from 6 weekold mice. Quantitative PCR was performed on a Step One Plus Real-time PCR system (Applied Biosystems, Carlsbad, CA, USA) using the SYBR Green PCR mixture (Roche Applied Science, Hague Road, IN, USA) as previously described ${ }^{60}$ : PCR primer sequences are listed in Supplementary Table 2. Transcript levels were normalized against $\beta$-actin expression. Relative values of the transcripts were derived using the equation, $2^{-\Delta \Delta C t}$, where $\Delta C t$ is equal to the difference in threshold cycles. Error bars were calculated as the range in fold change of VRK1-deficient transcript levels (mean \pm SEM) relative to the mean of normal transcript.

1. Matzuk, M. M. \& Lamb, D. J. The biology of infertility: research advances and clinical challenges. Nat Med 14, 1197-1213 (2008).

2. Kim, W. et al. Macro histone H2A1.2 (macroH2A1) protein suppresses mitotic kinase VRK1 during interphase. J Biol Chem 287, 5278-5289 (2012).

3. Kang, T. H. et al. Mitotic histone $\mathrm{H} 3$ phosphorylation by vaccinia-related kinase 1 in mammalian cells. Molecular and Cellular Biology 27, 8533-8546 (2007).

4. Kang, T. H., Park, D. Y., Kim, W. \& Kim, K. T. VRK1 phosphorylates CREB and mediates CCND1 expression. J Cell Sci 121, 3035-3041 (2008).

5. Lopez-Borges, S. \& Lazo, P. A. The human vaccinia-related kinase 1 (VRK1) phosphorylates threonine- 18 within the $\mathrm{mdm}-2$ binding site of the p53 tumour suppressor protein. Oncogene 19, 3656-3664 (2000).

6. Lancaster, O. M., Breuer, M., Cullen, C. F., Ito, T. \& Ohkura, H. The meiotic recombination checkpoint suppresses NHK-1 kinase to prevent reorganisation of the oocyte nucleus in Drosophila. PLoS Genet 6, e1001179 (2010).

7. Wiebe, M. S., Nichols, R. J., Molitor, T. P., Lindgren, J. K. \& Traktman, P. Mice deficient in the serine/threonine protein kinase VRK1 are infertile due to a progressive loss of spermatogonia. Biol Reprod 82, 182-193 (2010).

8. Choi, Y. H. et al. Vaccinia-related kinase 1 is required for the maintenance of undifferentiated spermatogonia in mouse male germ cells. PLoS One 5, e15254 (2010).

9. Schober, C. S., Aydiner, F., Booth, C. J., Seli, E. \& Reinke, V. The kinase VRK1 is required for normal meiotic progression in mammalian oogenesis. Mech Dev 128, 178-190 (2011).

10. Myers, M., Britt, K. L., Wreford, N. G., Ebling, F. J. \& Kerr, J. B. Methods for quantifying follicular numbers within the mouse ovary. Reproduction 127, 569580 (2004). 
11. Griffin, J., Emery, B. R., Huang, I., Peterson, C. M. \& Carrell, D. T. Comparative analysis of follicle morphology and oocyte diameter in four mammalian species (mouse, hamster, pig, and human). J Exp Clin Assist Reprod 3, 2 (2006).

12. Wang, Y. et al. Gonadotropin control of inhibin secretion and the relationship to follicle type and number in the hpg mouse. Biol Reprod 73, 610-618 (2005).

13. Allan, C. M. et al. Follicle-stimulating hormone increases primordial follicle reserve in mature female hypogonadal mice. J Endocrinol 188, 549-557 (2006).

14. Tilly, J. L. Ovarian follicle counts--not as simple as 1, 2, 3. Reprod Biol Endocrinol 1, 11 (2003).

15. Kerr, J. B. et al. Quantification of healthy follicles in the neonatal and adult mouse ovary: evidence for maintenance of primordial follicle supply. Reproduction 132 , 95-109 (2006)

16. Hwu, Y. et al. Imaging cells and tissues with refractive index radiology. Biophys J 87, 4180-4187 (2004).

17. Kim, J. et al. Altered branching patterns of Purkinje cells in mouse model for cortical development disorder. Sci Rep-Uk 1 (2011).

18. Donoghue, P. C. et al. Synchrotron X-ray tomographic microscopy of fossil embryos. Nature 442, 680-683 (2006).

19. Hwu, Y., Tsai, W. L., Groso, A., Margaritondo, G. \& Je, J. H. Coherence-enhanced synchrotron radiology: simple theory and practical applications. J Phys D Appl Phys 35, R105-R120 (2002).

20. Margaritondo, G., Hwu, Y. \& Je, J. H. Synchrotron light in medical and materials science radiology. Riv Nuovo Cimento 27, 1-40 (2004).

21. Boot, M. J. et al. In vitro whole-organ imaging: 4D quantification of growing mouse limb buds. Nat Methods 5, 609-612 (2008).

22. Megason, S. G. \& Fraser, S. E. Imaging in systems biology. Cell 130, 784-795 (2007)

23. Otoi, T., Fujii, M., Tanaka, M., Ooka, A. \& Suzuki, T. Canine oocyte diameter in relation to meiotic competence and sperm penetration. Theriogenology 54, 535$542(2000)$

24. Raghu, H. M., Nandi, S. \& Reddy, S. M. Follicle size and oocyte diameter in relation to developmental competence of buffalo oocytes in vitro. Reprod Fertil Dev 14, 5561 (2002).

25. Wang, X. N. \& Greenwald, G. S. Hypophysectomy of the cyclic mouse. I. Effects on folliculogenesis, oocyte growth, and follicle-stimulating hormone and human chorionic gonadotropin receptors. Biol Reprod 48, 585-594 (1993).

26. Danilovich, N. \& Sairam, M. R. Haploinsufficiency of the follicle-stimulating hormone receptor accelerates oocyte loss inducing early reproductive senescence and biological aging in mice. Biology of Reproduction 67, 361-369 (2002).

27. Matzuk, M. M., Burns, K. H., Viveiros, M. M. \& Eppig, J. J. Intercellular communication in the mammalian ovary: oocytes carry the conversation. Science 296, 2178-2180 (2002).

28. Eppig, J. J., Wigglesworth, K. \& Pendola, F. L. The mammalian oocyte orchestrates the rate of ovarian follicular development. Proc Natl Acad Sci U S A 99, 2890-2894 (2002).

29. Krisher, R. L. The effect of oocyte quality on development. J Anim Sci 82 E-Suppl, E14-23 (2004)

30. Sorensen, R. A. \& Wassarman, P. M. Relationship between growth and meiotic maturation of the mouse oocyte. Dev Biol 50, 531-536 (1976).

31. Otoi, T., Yamamoto, K., Koyama, N., Tachikawa, S. \& Suzuki, T. Bovine oocyte diameter in relation to developmental competence. Theriogenology 48, 769-774 (1997).

32. Fair, T., Hyttel, P. \& Greve, T. Bovine oocyte diameter in relation to maturational competence and transcriptional activity. Mol Reprod Dev 42, 437-442 (1995).

33. Yang, Y., Balla, A., Danilovich, N. \& Sairam, M. R. Developmental and molecular aberrations associated with deterioration of oogenesis during complete or partial follicle-stimulating hormone receptor deficiency in mice. Biol Reprod 69, 12941302 (2003)

34. Soyal, S. M., Amleh, A. \& Dean, J. FIGalpha, a germ cell-specific transcription factor required for ovarian follicle formation. Development 127, 4645-4654 (2000).

35. Behringer, R. R., Finegold, M. J. \& Cate, R. L. Mullerian-inhibiting substance function during mammalian sexual development. Cell 79, 415-425 (1994).

36. Durlinger, A. L. et al. Control of primordial follicle recruitment by anti-Mullerian hormone in the mouse ovary. Endocrinology 140, 5789-5796 (1999).

37. Juneja, S. C., Barr, K. J., Enders, G. C. \& Kidder, G. M. Defects in the germ line and gonads of mice lacking connexin43. Biol Reprod 60, 1263-1270 (1999).

38. Fortune, J. E. The early stages of follicular development: activation of primordial follicles and growth of preantral follicles. Anim Reprod Sci 78, 135-163 (2003).

39. Matsui, Y., Zsebo, K. M. \& Hogan, B. L. Embryonic expression of a haematopoietic growth factor encoded by the Sl locus and the ligand for c-kit. Nature 347, 667669 (1990).

40. Dong, J. et al. Growth differentiation factor-9 is required during early ovarian folliculogenesis. Nature 383, 531-535 (1996).

41. Elvin, J. A., Yan, C., Wang, P., Nishimori, K. \& Matzuk, M. M. Molecular characterization of the follicle defects in the growth differentiation factor 9deficient ovary. Mol Endocrinol 13, 1018-1034 (1999).
42. Dierich, A. et al. Impairing follicle-stimulating hormone (FSH) signaling in vivo: targeted disruption of the FSH receptor leads to aberrant gametogenesis and hormonal imbalance. Proc Natl Acad Sci U S A 95, 13612-13617 (1998).

43. Matzuk, M. M., Dionne, L., Guo, Q., Kumar, T. R. \& Lebovitz, R. M. Ovarian function in superoxide dismutase 1 and 2 knockout mice. Endocrinology 139, 4008-4011 (1998).

44. Ho, Y. S. et al. Reduced fertility in female mice lacking copper-zinc superoxide dismutase. J Biol Chem 273, 7765-7769 (1998).

45. Vainio, S., Heikkila, M., Kispert, A., Chin, N. \& McMahon, A. P. Female development in mammals is regulated by Wnt-4 signalling. Nature 397, 405-409 (1999).

46. Kano, K. et al. A novel dwarfism with gonadal dysfunction due to loss-of-function allele of the collagen receptor gene, Ddr2, in the mouse. Mol Endocrinol 22, 1866 1880 (2008).

47. Matsumura, H. et al. Transcriptome analysis reveals an unexpected role of a collagen tyrosine kinase receptor gene, Ddr2, as a regulator of ovarian function. Physiol Genomics 39, 120-129 (2009).

48. Krege, J. H. et al. Generation and reproductive phenotypes of mice lacking estrogen receptor beta. Proc Natl Acad Sci U S A 95, 15677-15682 (1998).

49. Lubahn, D. B. et al. Alteration of reproductive function but not prenatal sexual development after insertional disruption of the mouse estrogen receptor gene. Proc Natl Acad Sci U S A 90, 11162-11166 (1993).

50. Ogawa, S. et al. Roles of estrogen receptor-alpha gene expression in reproductionrelated behaviors in female mice. Endocrinology 139, 5070-5081 (1998).

51. Chen, L. M. et al. Subfertility with defective folliculogenesis in female mice lacking testicular orphan nuclear receptor 4. Mol Endocrinol 22, 858-867 (2008).

52. Smith, C. L. et al. Genetic ablation of the steroid receptor coactivator-ubiquitin ligase, E6-AP, results in tissue-selective steroid hormone resistance and defects in reproduction. Mol Cell Biol 22, 525-535 (2002).

53. Visser, J. A. \& Themmen, A. P. Anti-Mullerian hormone and folliculogenesis. Mol Cell Endocrinol 234, 81-86 (2005).

54. Castrillon, D. H., Miao, L., Kollipara, R., Horner, J. W. \& DePinho, R. A. Suppression of ovarian follicle activation in mice by the transcription factor Foxo3a. Science 301, 215-218 (2003).

55. Yan, C. et al. Synergistic roles of bone morphogenetic protein 15 and growth differentiation factor 9 in ovarian function. Mol Endocrinol 15, 854-866 (2001).

56. Brown, H. M. et al. ADAMTS1 cleavage of versican mediates essential structural remodeling of the ovarian follicle and cumulus-oocyte matrix during ovulation in mice. Biol Reprod 83, 549-557 (2010).

57. Sicinski, P. et al. Cyclin D2 is an FSH-responsive gene involved in gonadal cell proliferation and oncogenesis. Nature 384, 470-474 (1996).

58. Dierick, M., Masschaele, B. \& Van Hoorebeke, L. Octopus, a fast and user-friendly tomographic reconstruction package developed in LabView (R). Meas Sci Technol 15, 1366-1370 (2004)

59. Agresti, A. \& Franklin, C. Statistics: The Art and Science of Learning from Data, Edn. 2nd edition. (2007).

60. Woo, K. C. et al. Mouse period 2 mRNA circadian oscillation is modulated by PTB-mediated rhythmic mRNA degradation. Nucleic Acids Res 37, 26-37 (2009)

\section{Acknowledgments}

This work was supported by the Creative Research Initiatives (Functional X-ray Imaging) of MEST/NRF. K.T. Kim also acknowledges the supports from the National Research Foundation of Korea (NRF) (No. 20100030089).

\section{Author contributions}

J.K., K.T.K., and J.H.J. designed the research, J.K., Y.H.C., S.C., K.T.K., and J.H.J. conducted the research, J.K., Y.H.C. analyzed the data, J.K. and J.H.J. wrote the paper, J.K., Y.H.C., S.C., K.T.K., and J.H.J. edited the paper.

\section{Additional information}

Supplementary information accompanies this paper at http://www.nature.com/ scientificreports

Competing financial interests: The authors declare no competing financial interests.

License: This work is licensed under a Creative Commons

Attribution-NonCommercial-ShareAlike 3.0 Unported License. To view a copy of this license, visit http://creativecommons.org/licenses/by-nc-sa/3.0/

How to cite this article: Kim, J., Choi, Y.H., Chang, S., Kim, K.-T. \& Je, J.H. Defective folliculogenesis in female mice lacking Vaccinia-related kinase 1. Sci. Rep. 2, 468; DOI:10.1038/srep00468 (2012). 